\title{
A Note on the Coherence between Probability and Possibility Measures
}

\author{
Mamoni Dhar \\ Assistant Professor \\ Science College, Kokrajhar \\ Kokrajhar-783370, India \\ 1st \\ Author
}

\begin{abstract}
In this article, we intend to revisit the coherence established between possibility and probability from some functions which are density functions and would like to draw attention of the fact that since a possibility distribution of a normal fuzzy number can be expressed as two distribution functions by using set superimpositions, it seems that the efforts of finding the density functions which are possibility distributions and probability distributions at the same time would have no logical meaning from our standpoints so far.This paper also revisits the variable transformation established in accordance with some existing transformations. The aim of this paper is to contribute towards the development of a formal technique as well as methodological foundations that could deal with the outlined problems. A new procedure is proposed which disagree with all the existing principles. Further, logic behind our claim is put forward in details and it is expected that this would be able to satisfy all who are working to find possible consistency between possibility and probability.
\end{abstract}

\section{Keywords}

Randomness-Fuzziness Consistency Principle, GlivenkoCantelli's theorem, probability distribution.

\section{INTRODUCTION}

Possibility theory is a mathematical theory for dealing with certain types of uncertainty and is an alternative to probability theory. Possibility theory is an uncertainty theory devoted to the handling of incomplete information. As such, it complements probability theory. It differs from the latter by the use of a pair of dual set-functions (possibility and necessity measures) instead of only one. This feature makes it easier to capture partial ignorance. The process of converting possibility distribution into probability distribution and conversely has received attention in the past. This question is philosophically interesting as a part of debate between probability and fuzzy sets. The conversion problem between possibility and probability has its roots in the possibility probability consistency principle of Zadeh [16], that he introduced in the paper founding possibility theory in 1978. Dubious and Prade further contributed for its development. In Zadeh's view, possibility distributions were meant to provide a graded semantics to natural statements. However, possibility and necessity measures can also be the basis of a full-fledged representation of partial belief that parallels probability (Dubois and Prade 1988). Then, it can be seen either as a coarse, non-numerical version of probability theory, or as a framework for reasoning with extreme probabilities, or yet as a simple approach to reasoning with imprecise probabilities (Dubois, Nguyen and Prade, 2000).

Both probability and possibility may be seen as information about an experiment. It is conceivable to have at sometime that these are two forms of informations about some experiment and as a result the question of the relation between these two types of informations obtained arises at once. In this paper some aspect of concept the relationaship between these two are stated and it further explores the connection between this probability and possibility, which would in turn replace all other consistency principles established so far. This work also does not support the use of the term measure with possibility.

This relationship, however, seen differently by different researchers at different point of time in the literature of fuzzy set theory and the reader will find numerous approaches which from a mathematical point of view are quite interesting. At the same time, it is important to mention here that if so many consistency principles exist then it would be very difficult for one decide which principle to follow. In other words, it would be a time taking process for one to decide which to adopt and which to reject. This would obviously lead to a chaotic state. So we feel that is better to have a single intellectually satisfying principle instead of many having no logical foundation. We shall however mention a few of the existing consistency principles in this article.

\section{TRANSFORMATION CONSISTENCY PRINCIPLES:}

\subsection{Zadeh consistency principle:}

Zadeh defined the probability- possibility consistency principle such as "a high degree of possibilitydoes not imply a high degree of probability, nor does a low degree of probability imply a low degree of possibility" (Zadeh 1978). He defined the degree of consistency between a probability distribution $\mathrm{p}=\left(\mathrm{p}_{1}, \mathrm{p}_{2},, \mathrm{p}_{\mathrm{n}}\right) \ldots$ and a possibility distribution $\pi=\left(\pi_{1}, \pi_{2}, \ldots \ldots . \pi_{n}\right)$ as:

$$
C_{z}=\sum_{i=1}^{n} \pi_{i} \cdot p_{i}
$$

Zadeh pointed out that the probability -possibility consistency, defined in (1), is not a precise law or a relationship between possibility and probability distributions. It is an approximate formalization of the heuristic connection that a lessening of the possibility of an event tends to lessen its probability but not vice-versa (Zadeh 1978). From the above lines it is clear that Zadeh had some weaker constraints in mind. It is important to note here that in this process, Zadeh tried to define a probability law over the same space over which a possibility law has been defined and here the problem lies.

\subsection{Klir consistency principle:}

Klir was not satisfied with the Zadehian way of defining consistency between probability and possibility. He then tried to define it in his own way as seemed to him better than that 
defined earlier. The consistency principle proposed by Klir can be defined as follows:

Let $\mathrm{X}=\left\{w_{1}, w_{2}, \ldots \ldots w_{n}\right\}$ be a finite universe of singletons, let $p_{i}=p_{i}\left(w_{i}\right)$ and $\pi_{i}=\pi\left(w_{i}\right)$. Assume that the elements of $X$ are ordered in such a way that: $\forall i=$ $1 \ldots \ldots$. $p_{i}>0$ and $p_{i} \geq p_{i+1}$ and $\pi_{i}>0$ and $\pi_{i} \geq \pi_{i+1}$ with $p_{n+1}=0$ and $\pi_{n+1}=0$. According to Klir, the transformation from $p_{i}$ to $\pi_{i}$ must preserves some appropriate scale and the amount of information contained in each distribution (Klir 1993). The information contained in $\mathrm{p}$ or $\pi$ can be expressed by the equality of their uncertainties. Klir has considered the principle of uncertainty preservation under two scales:

- The ratio scale: This is a normalization of the probability distribution. The transformations

$p \rightarrow \pi$ and $\pi \rightarrow p$ are named the normalized

transformations and they are defined by

$$
\pi_{i}=\frac{p_{i}^{i}}{p_{1}} \text { and } p_{i}=\frac{\pi_{i}}{n \sum_{i=1}^{n} \pi_{i}^{i}}
$$

- The log-interval scales: the corresponding transformations $\mathrm{p} \rightarrow \pi$ and $\pi \rightarrow \mathrm{p}$ are defined by:

$$
\pi_{i}=\left(\frac{p_{i}^{i}}{p_{1}}\right)^{\alpha} \text { and } p_{i}=\frac{\pi_{i}^{\frac{1}{\alpha}}}{\sum_{i=1}^{n} \pi_{i}^{\frac{1}{\alpha}}}
$$

These transformations, which are named Klir transformations, satisfy the uncertainty preservation principle defined by (Klir 1993). $a$ is a parameter that belongs to the open interval ]0, 1 . In the way to satisfy uncertainty preservation principle, Klir tried to define a probability space, in the measure theoretic sense from the knowledge of possibilities concerned. Klir's transformation was debatable in the sense that the basic idea behind such developments can be thought of as the choice between possibility and probability remained a mere translation between languages. It appears that the principle was introduced only because there was the need of finding a relationship between probability and possibility and nothing else. This is clear because there is lack of logical foundation or mathematical framework in this principle.

\subsection{Dubois and Prade consistency principle:}

Dubios- Prade consistency principle came into force after Klir and Zadeh because they did not agree with the way of defining consistency between probability and possibility by their predecessors. Consequently, they developed another consistency principle in the assumption that it would overcome the shortcomings in the existing principles.This principle they stated in the following way:

The possibilistic representation is weaker than the probabilistic one because it explicitly handles imprecision (e.g. incomplete data) and because possibility measures are based on ordering structure than an additive one in the probability measures (Dubois 1993). Thus in going from a probabilistic representation to a possiblistic one, some information is lost because we go from point-valued probabilities to interval valued ones; the converse transformation adds information to some possibilistic incomplete knowledge.

The transformation $\pi \rightarrow p$ is guided by the principle of maximum specificity, which aims at finding the most informative possibility distribution. While the transformation $p \rightarrow \pi$ is guided by the principle of insufficient reason which aims at finding the probability distribution that contains as much uncertainty as possible but that retains the features of possibility distribution (Dubois 1993). This leads to write the consistency principle of Dubois and Prade such as:

$$
\mathrm{A} \subset \mathrm{X}: \Pi(\mathrm{A}) \geq \mathrm{P}(\mathrm{A})
$$

The transformations $p \rightarrow \pi$ and $\pi \rightarrow p$ are defined by:

$$
\begin{array}{r}
\pi_{i}=\sum_{j=1}^{n} p_{j} \text { And } \\
p_{i}=\sum_{j=1}^{n} \frac{\pi_{j}-\pi_{j+1}}{j}
\end{array}
$$

The two transformations defined by (2) and (3) are not converse of each other because they are not based on the same informational principle. For this reason, we name the transformation $\pi \rightarrow p$ defined by (3) as asymmetric one.

Dubois and Prade suggested a symmetric $p \rightarrow \pi$ transformation which is defined by:

$$
\pi_{i}=\sum_{j=1}^{n} \min \left(p_{i}, p_{j}\right)
$$

Dubois and Prade proved that the symmetric transformation $p \rightarrow \pi$, defined by (3), is the most specific transformation which satisfies the condition of consistency of Dubois and Prade defined by (4) (Dubois 1993). Thus here we can see the use of possibility measue. But the possibility measure is not a measure in the classical sense and so it can be said that the use of such words as possibility measure is not justifiable from our standpoints.

A possible justification of this may be stated as follows: The measure of a point is zero, Possibility of occurrence of a point is defined by membership function and therefore in this case the possibility of occurrence of the point is not zero. Hence there should not be any formalism with reference to the membership function. That is to say, the use of the word possibility measure is not justifiable from our standpoints of defining consistency principles.

These are the three most well- known consistency principles which can be found in the literature of fuzzy set theory from Zadeh's initial conception of possibility theory. These principles might have led many other authors of this field to develop many other principles of this kind without having any proper thinking that those principles defined probability over the same space over which possibility is defined. This is not the case with the transformation devised here. Another important thing to be noted here that all the well-known consistency principles that can be found in the literature of fuzzy set theory deal with the consistency in discrete case and nothing was discussed about the continuos cases. Continuos cases were discussed a bit in a paer of Dubios-Prade-Shandri but at the same time it was mentioned that these transformations were not related to each other and the converse transformations were shown to be inadequate. We 
shall however mention two more papers in which the authors had tried to find a relation in their way but in the process, they also committed the same mistake and thereby failed to define it properly. Due to this reason, we would like to draw attention in these principles.

\subsection{Some other related papers:}

Casreneira,Cubillo and Trillas [4] had tried to find a coherence of measures in a continuos iniverse when possibility and probability are determined by the same function.In other words, in order to find a coherence between possibility and probability measures in continuos case two things were considered. Firstly, some functions which are density functions were found and thereafter coherence between possibility and probability measures generated by the same density functions were introduced. It is to be noted here that in doing so the conditions which were applied to test whether a density function can be considered as a possibility distribution are as follows:

If $f: R \rightarrow[0,+\propto]$ is a bounded density function, then the function $\mu_{f}: R \rightarrow[0,+\propto]$ defined for each $\mathrm{x} \in R$ by

$$
\begin{aligned}
\mu_{f}(x) & =k f(k x) \\
\text { where } \quad k & =\frac{1}{\sup \{f(x), x \in R]},
\end{aligned}
$$

is a density function and a possibility distribution function.Additionally, if $\mathrm{f}$ is continuos , then there exists $y_{0} \in R$ such that $\mu_{f}\left(y_{0}\right)=1$.

Mouchaweb, Bouguelid, Biillaudel, RIERA [17], on the other hand, proposed a transformation from probability to possibility which they named as Variable transformation.This transformation is different from those proposed by Zadeh and Klir and was written as follows:

$$
\pi_{i}=\left(\frac{p_{i}}{p_{1}}\right)^{k\left(1-p_{i}\right)}
$$

Where $\mathrm{k}$ is a constant which gurantee the following condition of consistency:

$$
\forall w \in X: \pi(w) \geq p(w)
$$

This condition is a particular case of Dubious- Prade consistency principle but there is a condition that the value of ' $\mathrm{k}$ ' must belong to the following interval:

$$
0 \leq k \leq \frac{\log p_{n}}{\left(1-p_{n}\right) \cdot \log \frac{p_{n}}{p_{1}}}
$$

It was mentioned by them that this above mentioned transformation is different from Klir's transformation in the sense that Klir's transformation has a constant power $\alpha$ which belongs to the open interval $] 0,1\left[\right.$ while the power k.(1- $\left.p_{i}\right)$ in variable transformation, is a variable to make it more specific. So it can be said that the authors were in the opinion that the transformation proposed by Klir was not a specific one.

In this article, our intention is to show that all the above mentioned procedures are not preferable because we can show that a possibility space can be bifurcated into two probability spaces. In other words, a possibility distribution can be regarded as a combination of two probability distributions which are associated with some densities and hence it can be seen that the possibility distributions are related with density functions if procceded in our way and hence the type of conversions proposed by the said authors donot have some logical bearings. In other words, there is no need of converting the densities to possibility distributions in the way indicated since these are linked with each other in a natural way. Again the efforts to find the new transformations like variable transformation satisfying Dubious -Prade consistency principle is also not free from defects. It is because of the fact that there are some shortcomings in the principle on the basis of which variable transformation was derived. The reason for which we would like to discard the principle is stated clearly in earler section of this article. If this be the case then it is obvoius that any other principles which were developed on the basis of this would definitely not be workable. Here we would like to suggest some mathematical framework which may reduce the shortcomings in the existing consistency principles. For this we should have a glimse of DubiosPrade's definition of a normal fuzzy number because it is this definition which is taken as a base of expressing our views regarding consistency of possibility and probability. Dubious -Prade (see for example Kaufmann and Gupta (1984)) defined of a fuzzy number $X=[\alpha, \beta, \gamma]$ with membership function as:

$\mu_{X}(x)=\left\{\begin{array}{c}\psi_{1}(x), \alpha \leq x \leq \beta \\ \psi_{2}(x), \beta \leq x \leq \gamma \\ 0, \text { otherwise, }\end{array}\right.$

$\psi_{1}(x)$ being continous and non decreasing in the interval $[\alpha, \beta]$ and $\psi_{2}(x)$ being continuous and nonincreasing in the interval $[\beta, \gamma]$. Dubious- Prade definition is a firm step in defining fuzziness with the help of two different functions. In this article we would like use Dubious-Prade left reference function as a distribution function and similarly Dubios Prade left reference function as a complementary distribution function. Accordingly,we are interested in viewing $\psi_{1}(x)$ and $1-\psi_{2}(x)$ as probability distribution functions which would be associated with densities $\mathrm{d} \Psi_{1}(\mathrm{x}) / \mathrm{dx}$ and $\mathrm{d} 1-\Psi_{2}(x)$ $/ \mathrm{dx}$ and as such would like to consider the membership function of a normal fuzzy number from this perspective. In the process of establishing our claim we need the help of set superimpositions as defined by Baruah [3 \& 4] because whatever we do, we should do with proper mathematical frameworks to have a meaningful outcome and this framewok can be had from that of the operation of set superimposition.So before proceeding further let us have a look at the way in which the set superimposition is defined.

\section{THE OPERATION OF SET SUPERIMPOSITION:}

The operation of set superimposition is defined by Baruah [3\&4]. The possible justification of finding such an operation of superimposition can be as follows: When we overwrite, the overwritten portion looks darker. Indeed, in the overwritten portion there happens to double representation due to superimposition, which is why that portion looks darker. This happens due to superimposition. We now proceed to define this mathematically. The operation of set superimposition is expressed as follows: if the set $\mathrm{A}$ is superimposed over the set $\mathrm{B}$, we get 


$$
\mathrm{A}(\mathrm{S}) \mathrm{B}=(\mathrm{A}-\mathrm{B}) \mathrm{U}(\mathrm{A} \cap \mathrm{B})^{(2)} \cup(\mathrm{B}-\mathrm{A})
$$

where $S$ represents the operation of superimposition, and $(A \cap$ B) ${ }^{(2)}$ represents the elements of $(\mathrm{A} \cap \mathrm{B})$ occurring twice, provided that $(\mathrm{A} \cap \mathrm{B})$ is not void. With the application of superimposition of sets on uniformly fuzzy intervals, Baruah defined a normal fuzzy number of the type $N=[\alpha, b, \gamma]$ as

$$
\begin{gathered}
\mu_{\mathrm{N}}(\mathrm{x})=\Psi_{1}(\mathrm{x}), \text { if } \alpha \leq \mathrm{x} \leq \beta, \\
=\Psi_{2}(\mathrm{x}), \text { if } \beta \leq \mathrm{x} \leq \gamma, \\
\text { and } \quad=0 \text {,otherwise }
\end{gathered}
$$

where $\psi_{1}(x)$ and $1-\psi_{2}(x)$ are probability distribution functions which would be associated with densities $\mathrm{d} \Psi_{1}(\mathrm{x}) / \mathrm{dx}$ and $\mathrm{d} 1-\psi_{2}(x) / \mathrm{dx}$ and this would in turn lead to a very important principles which was named by Baruah [6] as the Randomness- Fuzziness Consistency Principle. In this article, we would like to take the help of this principle to attain our point of view. Let us have a look at this principle in short before proceeding further.

\section{THE RANDOMNESS -FUZZINESS CONSISTENCY PRINCIPLE:}

In this principle, it was stated that the existence of two laws of randomness is required to define a law of fuzziness. In other words, not one but two distributions with reference to two laws of randomness on two disjoint spaces can construct a fizzy membership function. This principle can be expressed mathematically in the following form:

For a normal fuzzy number of the type $N=[\alpha, \beta, \gamma]$ with membership function $\mu_{N}(x)=\Psi_{1}(x)$, if $\alpha \leq x \leq \beta,=\Psi_{2}(x)$, if $\beta$ $\leq x \leq \gamma$, and $=0$, otherwise, with $\Psi_{1}(\alpha)=\Psi_{2}(\gamma)=0, \Psi_{1}(\beta)=$ $\Psi_{2}(\beta)=1$, the partial presence of a value $x$ of the variable $X$ in the interval $[\alpha, \gamma]$ is expressible as $\mu_{N}(x)=\theta$ Prob $[\alpha \leq X \leq x]+(1-\theta)\{1-\operatorname{Prob}[\beta \leq X \leq x]\}$, where $\theta=1$ if $\alpha \leq \mathrm{x} \leq \beta \quad$ and $\theta=0$ if $\quad \beta \leq x \leq \gamma$

In other words, it can be said that with the help of two probability measures we can study possibility independently. It is to be mentioned here that with the introduction of this principle one can get rid of the effects of many existing principles leading to various results for the same thing. From the above mentioned consistency principle, we can say that the probability- possibility consistency principles which were derived over the same space have nothing to do. In other words, from the above it can be said that the consistency principles proposed by the authors at different point of time can never give us the results for which these were introduced. Hence care should be taken before proceeding further with these principles.

\section{CONCLUSIONS:}

In this article, efforts have been made to show that two laws of randomness are needed to define a normal fuzzy number with one law of randomness leading to the leading to the membership function on the left of the point of maximum possibility and another law of randomness leading to the membership function on the right of the point of maximum possibility. Since a possibility distribution of a normal fuzzy number can be expressed as two distribution functions by using set superimpositions, it seems that such types of efforts of finding the density functions which are possibility distribution and probability distributions at the same time would have no logical meaning from our standpoints. Since possibility distributions can be expressed either as a probability or as a complementary probability and hence these are already associated with some densities.For the same reason we would like to discard the variable transformation also.The result obtained by us wuth the help of operation of set superimposition seem more logical as it is established in accordance with the definitions of left reference function and right reference functions which are used to define a normal fuzzy number as can be found in the literature of fuzzy set theory. Finally we would like to say that the time has come to replace all the transformations which are found in the case of probability -possibility consistency with the one proposed by us because here every effort is made to make it logical and workable. Hence is our claim.

\section{REFERENCES}

[1] L.A. Zadeh, Inform. and Control, 338-353, 1965.

[2]Kaufman A. and M.M. Gupta,Introduction to Fuzzy Arithemetic, Theory and applications, Van Nostrand Reinhold Co. Inc., Wokingham, Berkshire, 1984

[3]Hemanta.K.Baruah, Set Superimposition and its Applications to the Theory of Fuzzy Sets, Journal of the Assam Science Society, Vol.40,No.1 \& 2, 25-31, 1999

[4] Hemanta.K.Baruah, Fuzzy Membership with respect to a Reference Function ,Journal of the Assam Science Society, Vol.40,No.3,1999,65-73.

[5] Elina Castineira,Susana Cubillo,Enric Trillas,On the Coherence between Probability and Posibility Measures,International Journal of Information Theories and Applications ,Vol. 14,303-310,2007

[6]Hemanta k. Baruah, the Randomness-Fuzzuness Consistency Principles, IJEIC, Vol. 1, Issue 1, 37-48, 2010

[7] Hemanta K. Baruah, Construction of the Membership Function of a Fuzzy Number, ICIC Express Letters, Vol. 5, Issue 2, 545-549, 2011.

[8] Hemanta. K .Baruah, Theory of Fuzzy sets Beliefs and Realities, IJEIC, Vol. 2, Issue 2, 1-22, 2011

[9] Hemanta K. Baruah, In Search of the Root of Fuzziness: The Measure Theoretic Meaning of Partial Presence, Annals of Fuzzy Mathematics and Informatics, Vol. 2, No. 1, 57 - 68, 2011.

[10] Hemanta K. Baruah, An Introduction to the Theory of Imprecise Sets: the Mathematics of Partial Presence, Journal of Mathematical and Computational Sciences, Vol. 2, No. 2, 2012, 110-124.

[11] Hemanta K. Baruah, Construction of Normal Fuzzy Numbers Using the Mathematics of Partial Presence, Journal of Modern Mathematics Frontiers, Vol.1, No. 1, 9 - 15, 2012.

[12] Dubious and Prade: Fuzy sets and Systems: Theory and Applications, Academic Press, New York (1980)

[13] Yamada.K. Probability-Posibility Transformation based on Evidence Theory, IEEE, 70-76

[14]Moamar Sayed mouchaweb, Mohamed Said Bouguelid, Patrice Billaudel, Bernard RiERA: variable ProbabilityPossibility transformation, 25 ${ }^{\text {th }}$ European Annual Conference on Human Decision -Making and Manual Control (EAM"06), September 27-29, Valenciennes, France.

[15]Didier Dubios, Laurent Foulloy, Gillis Mauris and Henry Prade: Probability - Possibility Transformations, Triangular Fuzzy Sets, and Probabilistic Inequalities.

[16] L. A. Zadeh: Fuzzy sets as a basis for a theory of possibility. Fuzzy Sets and System, 1, 3-28, 1978 